\title{
Reproductive dispersion and damping time scale with life-history speed
}

\author{
Sha Jiang ${ }^{1}$, Harman Jaggi ${ }^{1}$, WENYUN ZUO ${ }^{1}$, Madan Oli ${ }^{2}$, Tim Coulson ${ }^{3}$, Jean-Michel \\ Gaillard $^{4}$, and Shripad Tuljapurkar ${ }^{1}$ \\ ${ }^{1}$ Stanford University \\ ${ }^{2}$ University of Florida \\ ${ }^{3}$ Oxford \\ ${ }^{4}$ UMR-CNRS 5558
}

February 14, 2022

\begin{abstract}
Generation time has previously been the focus of comparative life history analyses. Here we examine three metrics: generation time $T_{c}$, reproductive dispersion $S$ (the distribution of ages of reproduction), and damping time $\tau$ (time to converge to stable (st)age distribution). We use data on 633 species of animals and plants, and perform phylogenetically corrected analyses. First we find that $S$ varies allometrically and isometrically with $T_{c}$. As a result, $\tau$ varies allometrically with either $T_{c}$ or $S$ but not both. Second, we find a trade-off between $\tau$ and $S$, so that $\tau$ does not vary isometrically with $T_{c}$. This trade-off is a novel demographic component to the relationship between $\tau, T_{c}$ and $S$ that is otherwise partly determined by their similarity as biological times. Our results indicate that species at the slow end of the slow-fast continuum take longer to converge to stable distribution than species with fast life-histories.
\end{abstract}

\section{Hosted file}

Reproductive dispersion and damping time scale with life-history speed.pdf available at https: //authorea.com/users/460440/articles/556454-reproductive-dispersion-and-dampingtime-scale-with-life-history-speed 